\title{
A Dialogue Game to Offer an Agreement to Disagree
}

\author{
Henk-Jan Lebbink \\ Computer Science \\ Utrecht/Radboud University \\ the Netherlands \\ henkjan@cs.uu.nl
}

\author{
Cilia Witteman \\ Social Sciences \\ Radboud University \\ the Netherlands \\ c.witteman@socsci.kun.nl
}

\author{
John-Jules Meyer \\ Computer Science \\ Utrecht University \\ the Netherlands \\ jj@cs.uu.nl
}

\begin{abstract}
This paper proposes a dialogue game in which coherent conversational sequences at the speech act level are described of agents that become aware they have an irresolvable disagreement and settle the dispute by agreeing to disagree. A disagreement is irresolvable from an agent's perspective if both agents are aware that they both ran out of options to resolve the situation. A dialogue game is formulated in which agents can offer information that may result in non-reconcilable, mutually inconsistent belief states. An agent's cognitive state consists of mental constructs, and given on these constructs, epistemic operators are defined which are used to define dialogue rules and a cognitive rule that allow an agent to agree to disagree.
\end{abstract}

\section{A Dialogue Game to Offer Information}

In general conversations, participating agents may have autonomy over their cognitive states, but they may also desire to change those of others. These agents may find themselves stuck in discussions about non-reconcilable beliefs.

In Beun [2] and Lebbink et al. [4] speech acts between agents are described and rules are identified that form a dialogue game that agents needs to play to fulfil their desires. A dialogue game consists of a set of dialogue rules that define which communicative acts an agent may utter given its current cognitive state. In addition, a dialogue game has a set of update rules that define the contents of the agent's cognitive state after a communicative act is uttered. In the current game we assume that information can only accumulate in the participants' cognitive states and cannot be retracted. In this information-monotonic approach additions may introduce inconsistent beliefs.

To define the agent's cognitive state allowing inconsistent beliefs we need a multi-valued logic (MVL). In MVL new truth-values are introduced to represent epistemic attitudes like unknown or inconsistent information states. In

Permission to make digital or hard copies of all or part of

this work for personal or classroom use is granted without fee

provided that copies are not made or distributed for profit or

commercial advantage and that copies bear this notice and the

full citation on the first page. To copy otherwise, to republish,

to post on servers or to redistribute to lists, requires prior

specific permission and/or a fee.

AAMAS'04, July 19-23, 2004, New York, New York, USA

Copyright 2004 ACM 1-58113-864-4/04/0007...\$5.00
Lebbink et al. [4], an MVL is introduced based on truthvalues from a bilattice structure. This allows us to create theories that are capable of being an agent's belief or ignorance state. A bilattice is an algebraic structure formalizing an intuitive space of generalized truth-values with two lattice orderings [3]. We use a bilattice for a four-valued logic by [1] in which truth-values $t$ and $f$ represent true and fal$\mathrm{se}$, and $\mathrm{u}$ and $\mathrm{i}$ represent a complete lack of information (unknown) and the inconsistent information state.

Theories of MVL logics are sets of propositions with certain properties. Three different theories are distinguished: normal theories which represent the agent's desires; complete theories representing the agent's belief state; and dual theories representing the agent's ignorance state. If an agent believes a that term $p$ has at least truth-value t, i.e. $p: t$, and it also concludes to believe $p: \mathrm{f}$, it also believes $p: \mathrm{i}$ stating that the agent has an inconsistent belief state with regard to $p$.

The agent's cognitive state consists of a finite number of mental constructs which are theories of MVL. We will not present a formal definition of the different epistemic operators like belief, desire and ignorance that agents have regarding themselves and other agents; it suffices to say that the agent has private mental constructs and it keeps track of mental constructs of others in manifested mental constructs.

Cognitive processes are described with rules that state when an agent is allowed to add a proposition to its belief state. These rules apply when their criteria hold in the agent's cognitive state. Three cognitive capabilities or processes are distinguished: deciding to believe propositions, deducing consequences of newly accepted beliefs, and deducing that one has an irresolvable disagreement with another agent. We assume that agents are very credulous: they accept to believe a proposition if they are aware that another agent believes the proposition and it is consistent with their current belief state on a first-come-first-serve basis.

If we are to specify the semantics of communicative acts, we are to give the criteria for uttering the acts, and the consequences these acts have on the agent's cognitive state. A 
dialogue rule for a speech act $\lambda$ states that if all criteria of $\lambda$ hold according to an agent's cognitive state, then $\lambda$ may be uttered. Update rules define the contents of an agent's cognitive state after a communicative act is uttered. An update rule for a speech act $\lambda$ states that if $\lambda$ is directed at an agent, then the post-conditions of $\lambda$ hold for that agent. Dialogue and update rules define the space of all possible dialogues in the dialogue game.

An agent's motivation to utter a question can be described when it is to balance its belief and desire state [2]. In a similar fashion the motivation to offer information regarding some proposition $p: \theta$ is described as the situation in which an agent $x$ has the desire that $y$ is to believe $p: \theta$, and $x$ is not aware that $y$ already believes $p: \theta$. The full set of criteria and post conditions of offers and answers to offers are not presented due to space limitations here.

\section{To Agree to Disagree}

If two agents are unable to agree on a belief, a persuasion dialogue, similar to the dialogue game to offer information, may resolve the disagreement by adding information to the agents' belief states [5]. If all methods to persuade are exhausted, the agents can conclude that they disagree on a specific proposition and that they both agree on this.

An agent $x$ is aware that it disagrees with agent $y$ about the truth-value of proposition $p$ iff $x$ believes $p: \theta_{1}$ and $x$ believes that $y$ believes $p: \theta_{2}$, and the truth-values are in disagreement, which in a four-valued logic can occur in only one possibility: $t$ disagrees with $f$. A second-order disagreement awareness exists when an agent $x$ is aware that another agent $y$ believes a proposition $p: \theta_{1}$ and $x$ is aware that $y$ is aware that $x$ believes proposition $p: \theta_{2}$ and the truth-values are in disagreement.

The cognitive rule that states when an agent may believe that it has an irresolvable disagreement can now be captured in a reasoning rule. An agent $x$ may add to its belief state an agreement to disagree with agent $y$ about term $p$ (we call agent $x$ 'I' and agent $y$ 'you'):

1. I am aware that I have a disagreement with you about term $p$.

2. I am aware that you are also aware of the disagreement.

3. There is no set of propositions that I believe and that I have not offered to you that could have resolved the disagreement if you had accepted to believe them. In this situation, I have no methods (sets of propositions) left to persuade you.

4. I am aware that there is no set of propositions that you believe and that you have not offered to me before that could have resolved the disagreement if I had accepted

Permission to make digital or hard copies of all or part of this work for personal or classroom use is granted without fee provided that copies are not made or distributed for profit or commercial advantage and that copies bear this notice and the full citation on the first page. To copy otherwise, to republish, to post on servers or to redistribute to lists, requires prior

specific permission and/or a fee.

AAMAS'04, July 19-23, 2004, New York, New York, USA

Copyright 2004 ACM 1-58113-864-4/04/0007...\$5.00 to believe them. In this situation, I think that you have no methods (sets of propositions) left to resolve the situation.

If these four criteria hold then the agent is allowed to believe that it is stuck in an irresolvable disagreement. The dialogue game takes care of communicating this information to agent $y$ and $y$ reaching the actual agreement, making the agreement to disagree common belief.

\section{Conclusions}

What is lacking in Beun [2] and Lebbink et al. [4] is the possibility for agents to recognize an irresolvable disagreements and, based on this recognition, to offer an agreement to disagree. In this paper we have given the sketch of a formal semantics for a dialogue game that enables agents to offer information, and we have given the criteria to become aware of an irresolvable disagreement, which can be communicated with the dialogue game, making the disagreement common belief. This common belief may motivate dialogues to weaken arguments in order to retract propositions that would resolve the disagreement, or to redefine the meaning of propositions that resulted in the disagreement.

Dialogues can be generated from the dialogue and update rules, providing the possibility to analyse dialogue games on useful properties like termination, whether the unbalanced desire/belief states are resolved in the agent's cognitive state if the dialogue has ended, or whether the dialogue ends in uniqueness of cognitive states (confluence). The presented game is not confluent because an agent accepts to believe a proposition if it is consistent with its current belief state. This makes the timing of communicative acts of crucial importance, resulting in the possibility of different end states.

\section{References}

[1] N. D Belnap Jr. A useful four-valued logic. In J. Michael Dunn and G. Epstein, editors, Modern Uses of Multiple-Valued Logic, pages 8-37, Dordrecht, 1977. D. Reidel.

[2] Robbert-Jan Beun. On the Generation of Coherent Dialogue: A Computational Approach. Pragmatics \& Cognition, 9(1):37-68, 2001.

[3] Matthew L. Ginsberg. Multivalued Logics: A Uniform Approach to Reasoning in Artificial Intelligence. Computational Intelligence, 4:265-316, 1988.

[4] Henk-Jan Lebbink, Cilia L.M. Witteman, and John-Jules Ch. Meyer. Dialogue Games for Inconsistent and Biased Information. Electronic Lecture Notes of Theoretical Computer Science, 52(2), June 292003.

[5] Douglas N. Walton and E. C. W. Krabbe. Commitment in Dialogue: Basic Concepts of Interpersonal Reasoning. SUNY Press. Albany, NY, USA, 1995. 